\title{
Searching for Cosmic-Ray Signal from Decay of Fermionic Dark Matter with CALET
}

\author{
Saptashwa Bhattacharyya ${ }^{* a}$, Holger Motz $^{b}$, Shoji Torii ${ }^{a, c}$, Yoichi Asaoka ${ }^{c}$ \\ ${ }^{a}$ Graduate School of Advanced Science and Engineering, Waseda University \\ ${ }^{b}$ International Center for Science and Engineering Programs, Waseda University \\ ${ }^{c}$ Research Institute for Science and Engineering, Waseda University \\ E-mail: saptashwaberuri.waseda.jp
}

\begin{abstract}
The ISS-based CALET detector which is in operation since October 2015, can play an important role in indirect search of Dark Matter by measuring the electron + positron cosmic-ray spectrum in the $\mathrm{TeV}$ region for the first time directly. With its fine energy resolution $(\sim 2 \%)$ and high proton rejection ratio $\left(1: 10^{5}\right)$, CALET has a capability to detect fine structures in $\left(e^{+}+e^{-}\right)$ spectrum. In this work, we have investigated CALET's potential to discern between Dark Matter decay and nearby pulsars as the origin of the Cosmic Ray positron excess observed by PAMELA or AMS-02. A parametrization of the propagated electron and positron spectra is fitted to the existing measurements, where either 3-body decay of Fermionic Dark Matter or pulsar assumed responsible for the positron excess. Expected CALET data for Dark Matter decay models which can explain the positron excess are calculated and analyzed. The signal from a particular 3-body Dark Matter decay, which can explain the measurements from the AMS-02 experiment, is shown to be distinguishable from a single pulsar source by observing $\left(e^{+}+e^{-}\right)$spectrum with CALET. We show that an especially clear separation of the pulsar model is possible from the Dark Matter model for which the diffuse $\gamma$-ray flux is possibly compatible with the Fermi-LAT data.
\end{abstract}

35th International Cosmic Ray Conference - ICRC2017

10-20 July, 2017

Bexco, Busan, Korea

\footnotetext{
* Speaker.
} 


\section{Introduction}

AMS-02 confirmed an increase in the positron fraction above $10 \mathrm{GeV}$ [1] with good statistics, which was observed initially by PAMELA [2]. This rise of the positron fraction suggests the presence of an extra source emitting electron-positron pairs, such as, pulsars or decay and annihilation of Dark Matter (DM) [3]. Since the flux from DM decay scales with the decay rate, it can naturally explain the positron excess, in contrast to DM annihilation models which require a boost factor $[4,5]$. Among different DM decay models, leptonic decay avoids anti-proton overproduction, and 3-body decay is favorable for the explanation of the positron excess compared to 2-body decay as it produces a softer positron spectrum, which is consistent with the present data.

In this work, we investigated the possibility of discerning such a signal of a DM decay from a single pulsar source by using the $\left(e^{+}+e^{-}\right)$spectrum being measured by the CALorimetric Electron Telescope (CALET). CALET, currently in operation on the ISS, searches for signatures of nearby CR sources and/or DM in the $\left(e^{+}+e^{-}\right)$spectrum with its fine energy resolution $(2 \%)$ and high proton rejection power $\left(1: 10^{5}\right)[6,7]$.

We study a DM candidate with a mass of $1-2 \mathrm{TeV}$, decaying to a lepton-anti-lepton pair and a neutrino, as a possible extra source which can explain the positron excess [8]. We parametrize the propagated electron and positron spectra, and calculate the $\left(e^{+}+e^{-}\right)$spectrum expected to be measured by CALET, assuming DM decay to be the extra source. We fit the same parametrization with a single pulsar source to the simulated CALET data to evaluate CALET's ability to discern a pulsar from this DM decay.

The decay of DM is accompanied by $\gamma$-ray emission, which is compared with the Fermi-LAT diffuse $\gamma$-ray flux measurement [9]. We show that $\gamma$-ray over-production can be reduced significantly for the chosen DM model, depending on the branching ratios of the charged primary decay products. We show that the DM model with low $\gamma$-ray yield has a signature which is especially well detectable by the CALET measurement. Models of this kind are of high importance in the indirect DM search with CALET.

\section{Three Body Dark Matter Decay and Decay Spectrum}

To explain the positron excess with a 3-body decay of DM, we investigate a theoretical model where the Standard Model (SM) is extended by 3 fermionic singlets $(N, \psi, S)$ and two Higgs doublets $(\eta, \chi)[8]$, with the lightest fermion $N$ as the DM candidate.

In our parametrization of this model, the inverse of the decay times $\left(\frac{1}{\tau_{e}}, \frac{1}{\tau_{\mu}}, \frac{1}{\tau_{\tau}}\right)$ of the DM for the individual channels $(N \rightarrow e e v, N \rightarrow \mu \mu \nu, N \rightarrow \tau \tau v)$ are the free parameters. The decay of the DM is mediated by heavy scalars $\eta, \chi\left(\sim 10^{3} \mathrm{TeV}\right)$ with negligible lifetime, making 4-point scalar interaction a good approximation for the DM decay process. With this assumption the probability distribution for the momentum of the charged leptons $\left(e^{+} e^{-}, \mu^{+} \mu^{-}, \tau^{+} \tau^{-}\right)$is given by

$$
\frac{1}{\Gamma} \frac{d \Gamma}{d x}=2 x^{2}(3-2 x)
$$

where $x=E / E_{\max }$ and $E_{\max }=0.5 \mathrm{M}_{\mathrm{DM}}$.

From this initial energy distribution, the $e^{+}$and $e^{-}$spectra produced per decay, $\frac{d N}{d E}$, is calculated using the event generator PYTHIA (Version 8.2) [10]. The spectra for $e^{+}$and $e^{-}$are identical, 
and the $e^{+}$spectrum is propagated in GALPROP [11] within the galaxy, in a diffusion zone of size $16(X) \times 16(Y) \times 6(Z) \mathrm{kpc}^{3}$. The spatial diffusion coefficient given by $D_{x x}=D_{0}\left(\frac{R}{R_{0}}\right)^{\delta}$ is normalized to $D_{0}=2.9 \times 10^{28} \mathrm{~cm}^{2} \mathrm{~s}^{-1}$, at a rigidity $R_{0}=4 \mathrm{GV}$. We choose $\delta=0.4$, and an Alfven speed of $12 \mathrm{~km} \mathrm{~s}^{-1}$ for this propagation calculation. We assume a Navarro-Frenk-White (NFW) profile [12] for the DM density $\left(\rho_{\mathrm{DM}}\right)$ in our galaxy to calculate the injected particles per volume and time, given by

$$
Q=\Gamma \frac{\rho_{\mathrm{DM}}}{\mathrm{M}_{\mathrm{DM}}} \frac{d N}{d E},
$$

where $\Gamma$ and $\mathrm{M}_{\mathrm{DM}}$ are decay rate and mass of the DM, respectively.

\section{Parametrization of $e^{+}+e^{-}$Flux and Fit to Positron Excess}

We parametrize the propagated $e^{+}$and $e^{-}$spectra to reflect the variability of the free parameters of injection and propagation processes. We apply this parametrization to determine different models with DM as the extra source by fitting to the $\left(e^{+}+e^{-}\right)$and the positron flux measured by AMS-02 [13, 14]. For this parametrization, we assume distant supernova remnants (SNR) as the primary sources, giving a power law spectrum, to which a secondary component from the interaction of CRs with the interstellar medium (ISM) is added. For the primary and the secondary flux we assume $\gamma_{p}, \gamma_{s}$ to be the power law indices and $C_{p}, C_{s}$ to be the absolute normalizations, respectively. The effect of radiative energy loss processes during propagation of primary electrons is modeled as an exponential cut-off at energy $E_{d}$. This term is omitted for secondary particles due to them propagating a shorter distance compared to primaries. With these parameters, the $\left(e^{+}+e^{-}\right)$ and the positron-only flux can be written as

$$
\phi_{T}=2 \phi_{\text {extra }}+C_{p} E^{\gamma_{p}}\left(2 \frac{C_{s}}{C_{p}} E^{\gamma_{s}-\gamma_{p}}+e^{\left(\frac{-E}{E_{d}}\right)}\right) \quad, \quad \phi_{e^{+}}=\phi_{\text {extra }}+C_{s} E^{\gamma_{s}},
$$

respectively, where $\phi_{\text {extra }}$ is the flux from the extra source emitting electron-positron pairs.

The extra source flux from the decay of DM is given by

$$
\phi_{\mathrm{DM}}=\frac{1}{\tau_{e}} \phi_{e}+\frac{1}{\tau_{\mu}} \phi_{\mu}+\frac{1}{\tau_{\tau}} \phi_{\tau},
$$

with $\phi_{e}, \phi_{\mu}, \phi_{\tau}$ being the $e^{+}$(identical to $e^{-}$) spectra for decay to $e e v, \mu \mu \nu, \tau \tau v$, respectively.

For the pulsar model we parametrize the extra source by

$$
\phi_{p n}=C_{p n} E^{\gamma_{p n}} e^{-\left(\frac{E}{E_{p n}}\right)},
$$

where $C_{p n}$ is the normalization of the spectrum, which is defined by the power law index $\gamma_{p n}$, and the cut-off energy $E_{p n}$.

The above parametrization of $\left(e^{+}+e^{-}\right)$and positron flux with either the DM or pulsar as the extra source is fitted to the present measurements by AMS-02, in order to determine values for the free parameters. We show an example fitting result with $2 \mathrm{TeV} D M$ as the extra source in figure 1a, where the spectrum matches best with measurements for a branching ratio of 0.77 to $\tau \tau \nu$ and 0.23 to $e e v$. This type of spectrum is characterized by a rapid decrease of the $\left(e^{+}+e^{-}\right)$and positron flux at approximately half the DM mass. This spectral feature of DM as the extra source is considerably 
different from a smooth spectrum as shown figure $1 \mathrm{~b}$, where the parametrization with a pulsar as the extra source is fitted to AMS-02 measurements, with $E_{p n}$ fixed to $1 \mathrm{TeV}$.

The upper bound of the fit range is $1 \mathrm{TeV}$, as there are no high resolution data points from AMS- 02 measurements above $1 \mathrm{TeV}$. Therefore, the parameter $E_{d}$ cannot be determined by fitting and was set to a fixed value of $2 \mathrm{TeV}$ in both these fits. To estimate the unknown $\mathrm{TeV}$ region of the $\left(e^{+}+e^{-}\right)$spectrum, the contribution from the most influential nearby source [15], the Vela SNR, is calculated using GALPROP. To consider the effect of the Vela SNR in the CR $\left(e^{+}+e^{-}\right)$spectrum, several values ( $1 \mathrm{TeV}, 2 \mathrm{TeV}, 5 \mathrm{TeV}, 10 \mathrm{TeV}$ ) for $E_{d}$ are studied.

The lower bound of the fit is $15 \mathrm{GeV}$, as the $\mathrm{CR}$ spectra below $15 \mathrm{GeV}$ is influenced by solar modulation, diffusive reacceleration, and possibly a break in the injection index which are difficult to represent by a simple parametrization. To include the effects of solar modulation above $15 \mathrm{GeV}$, the force field approximation is applied with $500 \mathrm{MeV}$ for the potential.
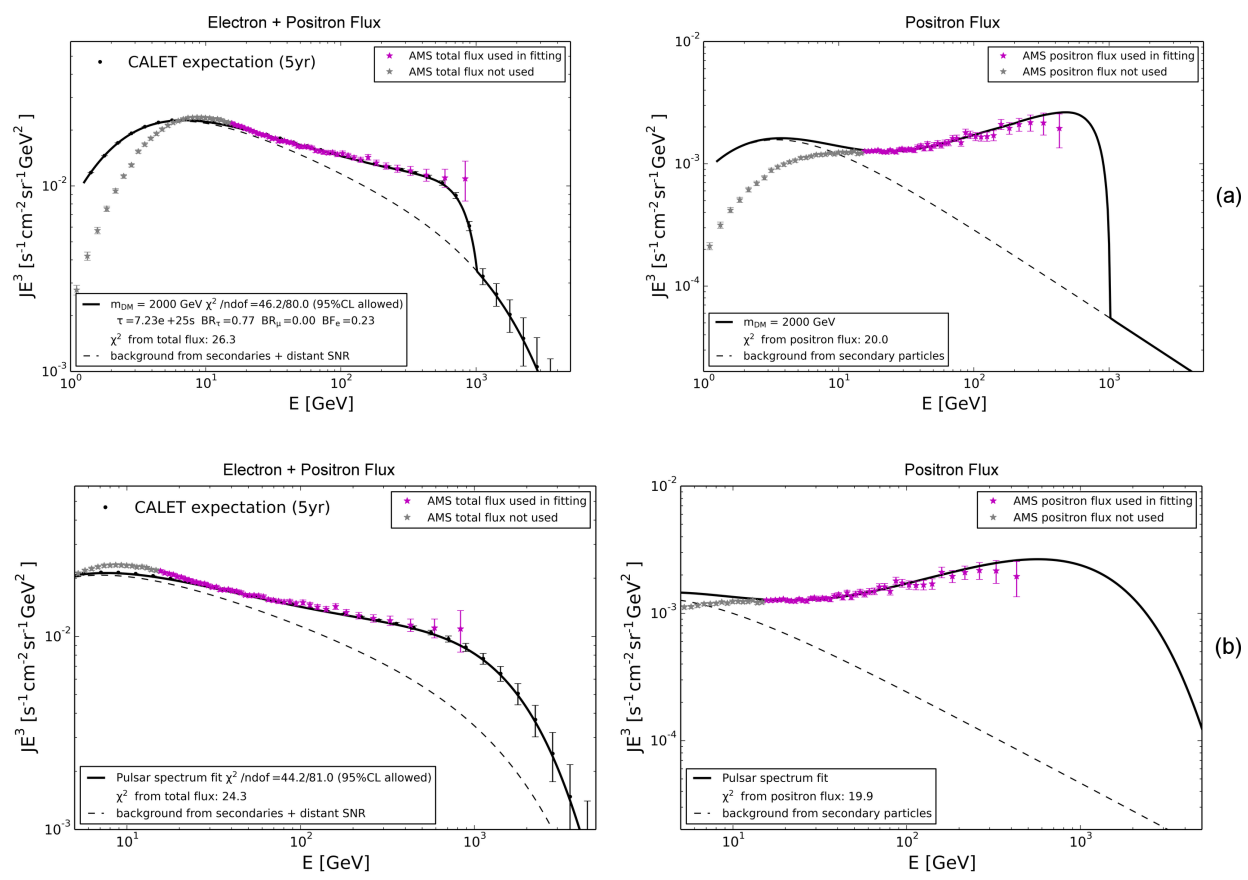

Figure 1: (a) $2 \mathrm{TeV}$ DM decay spectra on top of the background (dotted line) are fitted to the positron flux and $\left(e^{-}+e^{+}\right)$flux from AMS-02 resulting in a branching ratio of 0.77 to $\tau \tau \nu$ and 0.23 to eev channel, with $E_{d}=2 \mathrm{TeV}$. (b) Similarly, the single pulsar parametrization on top of the background is fitted with the AMS-02 $\left(e^{-}+e^{+}\right)$and positron flux with fix cut-off energies for background $\left(E_{d}=2 \mathrm{TeV}\right)$ and pulsar $\left(E_{p n}=1 \mathrm{TeV}\right)$.

\section{Diffuse $\gamma$-ray Measurement and Low $\gamma$-ray Model}

The decay of the DM is accompanied by $\gamma$-ray production by Final State Radiation (FSR), decay of the primary decay products and by inverse Compton scattering (ICS) during propagation of the charged decay products. From these processes we expect a diffuse $\gamma$-ray flux in the galactic DM-halo. Even though the largest concentration of DM is in the central region of Milky-Way, the 
$\gamma$-rays from the astrophysical sources provide a large background in this region, and due to this we concentrate on higher latitudes $\left(|b|>20^{\circ}\right)$ for comparison of the $\gamma$-ray flux from DM decay with Fermi-LAT data. The diffuse $\gamma$-ray flux in this region is so far available only from Fermi-LAT, currently operating detectors with thicker calorimeters such as CALET $\left(30 X_{0}\right)$ [6], will provide data to confirm this measurement.

The $\gamma$-ray flux from the DM decay depends on both the mass of the decaying DM and on the decay products. Among the different decay channels $(e e v, \mu \mu \nu, \tau \tau \nu), \tau \tau \nu$ causes the largest $\gamma$-ray production. To study the possibility of a DM-only explanation of the positron excess compatible with the current $\gamma$-ray measurements, the branching ratio of the $\tau \tau \nu$ channel is reduced. This is possible in the chosen DM model, since the branching ratios of the outgoing leptons are completely free parameters. Starting with the parameters obtained from the initial fit, $\frac{1}{\tau_{\tau}}$ is reduced in steps while adapting all other free parameters in each step, until the $\chi^{2}$ of either positron flux or $\left(e^{+}+e^{-}\right)$ flux exceeds $95 \% \mathrm{CL}$, or $\frac{1}{\tau_{\tau}}$ reaches zero.

We show in figure 2 the $\gamma$-ray fluxes from the different components for two different DM models. $\gamma$-ray flux from the decay of $2 \mathrm{TeV} \mathrm{DM}$ decaying to $\tau \tau \nu(73 \%)$ and eev (27\%) as introduced in section 3, is compared with a low $\gamma$-ray flux DM model, where a $1 \mathrm{TeV} \mathrm{DM}$, decaying only to $\mu \mu v(60 \%)$ and eev (40\%) channel explains the positron excess according to the procedure explained above, resulting in the spectrum as shown in figure 3. $\gamma$-ray flux from the decay of the charged primaries $\left(e^{ \pm}, \mu^{ \pm}, \tau^{ \pm}\right)$and FSR are calculated using PYTHIA assuming NFW profile. The secondary $\gamma$-ray production, which is caused by interaction of the decay products with

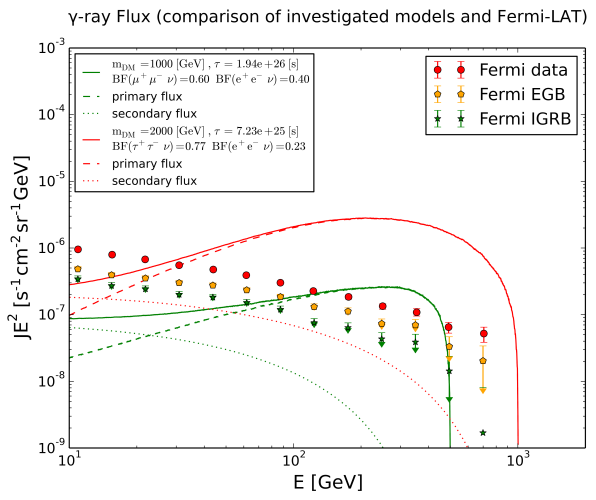

Figure 2: Predicted $\gamma$-ray flux from decay of $2 \mathrm{TeV}$ (red line) and $1 \mathrm{TeV}$ DM (green line) are compared to Fermi-LAT diffuse $\gamma$-ray measurement. The total $\gamma$-ray flux including the primary (dash dot line) and secondary production (dotted line) are shown for both $2 \mathrm{TeV}$ and $1 \mathrm{TeV}$ DM models. the interstellar radiation field (ISRF) is calculated using GALPROP, averaging over the flux at latitudes $|b|>20^{\circ}$. While the $2 \mathrm{TeV}$ DM model produces a $\gamma$-ray flux which clearly exceeds the the Fermi-LAT data, $\gamma$-ray flux from the $1 \mathrm{TeV} \mathrm{DM}$ model (decay excluding $\tau \tau \nu$ channel) is close to the experimental data.

In this fermionic DM model, DM with low mass and no decay to $\tau \tau \nu$ channel case is least in conflict with the Fermi-LAT $\gamma$-ray measurement. Even though the predicted $\gamma$-ray flux from the $1 \mathrm{TeV} \mathrm{DM}$ is somewhat higher than the Fermi-LAT measurement, there should be an uncertainty in the lifetime of the DM, and thus the $\gamma$-ray flux, from the choice of propagation conditions used for the positrons of the DM decay. Also the shape of the DM halo may influence the charged $\mathrm{CR}\left(e^{+}, e^{-}\right)$and $\gamma$-ray flux. The $\gamma$-ray flux measured at higher latitudes may be reduced and the charged CR flux enhanced if the DM accumulates close to galactic plane, as in the "Dark-Disc" model [16] for partly self-interacting DM. 

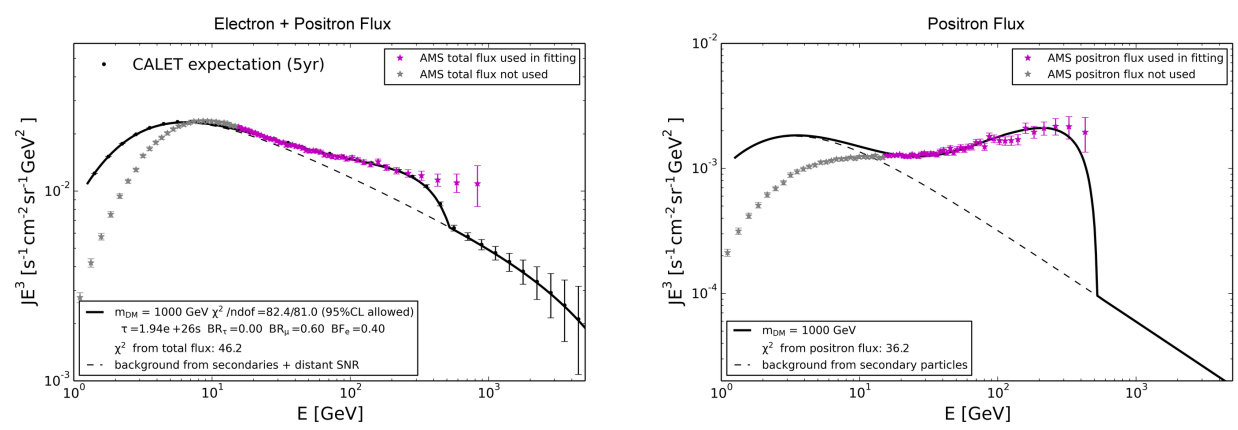

Figure 3: $1 \mathrm{TeV}$ DM decay spectra (excluding $\tau \tau \nu$ channel) on top of the background (dotted line) are fitted to the $\left(e^{-}+e^{+}\right)$flux (left) and positron flux (right) measured by AMS-02, resulting in a branching ratio of 0.60 to $\mu \mu v$ and 0.40 to eev channel. $E_{d}$ is fixed to $10 \mathrm{TeV}$.

\section{CALET Capability to Discern Signals from DM Decay and Single Pulsar Source}

To study CALET's capability to distinguish a signal of the decay of fermionic DM from a single pulsar source, we calculate CALET expected data for the fits of the parametrization with the DM as extra source to the experimental results, based on the detector's aperture of $1200 \mathrm{~cm}^{2} \mathrm{sr}$ [17] and data-taking for 5 years with a reconstruction efficiency of $90 \%$. To simulate the statistical fluctuations in the event rates, $10^{3}$ event samples were generated, representing different outcomes of the $\left(e^{+}+e^{-}\right)$flux measurement in each of the DM decay models. The energy spectrum from one of these samples for the $2 \mathrm{TeV}$ DM model is shown in figure 4a. Then, the single pulsar source parametrization was fitted to the simulated 5-year $\left(e^{+}+e^{-}\right)$flux CALET data for all the generated samples for the DM and the positron flux measured by AMS-02, giving a $\chi^{2}$ distribution. In this fit the free parameters for the pulsar model are $\frac{C_{p n}}{C_{p}}, \gamma_{p n}-\gamma_{p}, E_{p n}$, and for the background are $C_{p}, \frac{C_{s}}{C_{p}}, \gamma_{p}, E_{d}$. The DM model is refitted to the same data points to obtain a $\chi^{2}$ distribution for the DM, and finally these two $\chi^{2}$ distributions are compared to discern between DM decay and single pulsar model. As a part of our low $\gamma$-ray of DM decay study, we follow the same steps and equivalent fit of single pulsar to a case sample for $1 \mathrm{TeV}$ DM model (decay excluding $\tau \tau \nu$ channel) is shown in figure $4 \mathrm{~b}$.

The $\chi^{2}$ distribution as shown in the left panel of figure 5 is obtained from fitting the single pulsar source to the 5-year CALET data for the $10^{3}$ simulated samples of a $2 \mathrm{TeV}$ DM (for the $\left(e^{+}+e^{-}\right)$flux $)$and positron flux from the AMS-02 measurement. This is compared with the $\chi^{2}$ distribution from re-fitting the DM model to these same data points. Similarly, $\chi^{2}$ distribution plot for $1 \mathrm{TeV} \mathrm{DM}$ decay model is also shown in the right panel of figure 5 .

Since both the extra source models (DM and pulsar) have same number of free parameters, we use Akaike Information Criterion [18] to select one model over another by qualitative separation. Comparing the two $\chi^{2}$ distribution plots for $2 \mathrm{TeV}$ and $1 \mathrm{TeV}$ DM model, the $1 \mathrm{TeV}$ DM case features largest difference between the $\chi^{2}$ distribution for pulsar and DM model. This is shown in the right panel of figure 5, which demonstrates, that this $1 \mathrm{TeV}$ DM model is more favorable to discern from a single pulsar, compared to the $2 \mathrm{TeV}$ DM model, by the CALET $\left(e^{+}+e^{-}\right)$flux measurement. This $1 \mathrm{TeV}$ DM case is least in conflict with the Fermi-LAT $\gamma$-ray measurement, 

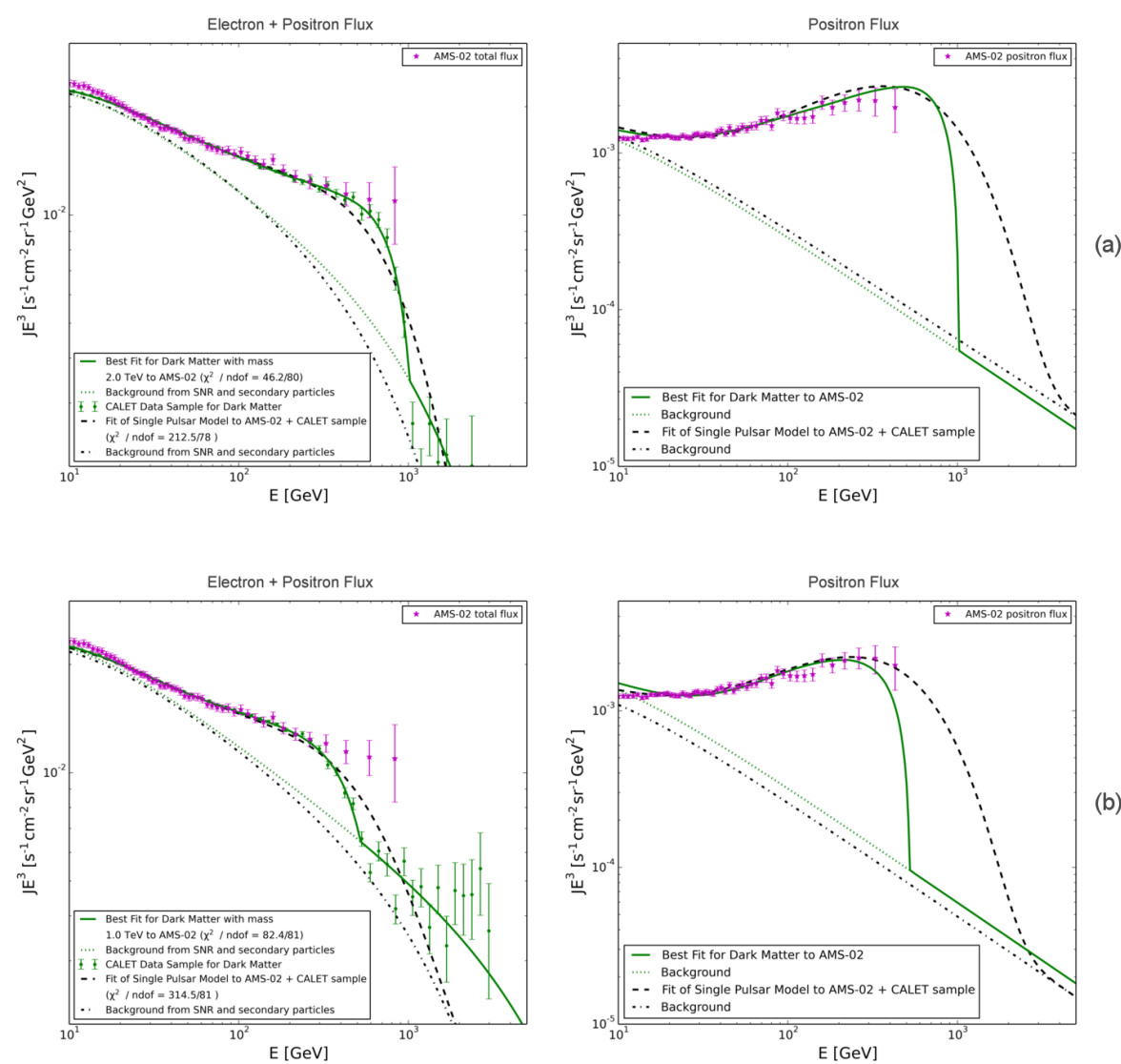

Figure 4: (a) Fit of the single pulsar source to one of the simulated $10^{3}$ statistical samples (green line) of 5 year CALET data for $\left(e^{+}+e^{-}\right)$flux (left) for the $2 \mathrm{TeV}$ DM decay model and AMS-02 positron flux (right), is shown here with black dashed line, assuming $E_{d}=2 \mathrm{TeV}$. Background spectra for two different models are shown with the dotted lines (green and black for DM and pulsar respectively). (b) As figure $4 \mathrm{a}$, but for $1 \mathrm{TeV} \mathrm{DM}$ model (decay excluding $\tau \tau \nu$ channel), assuming $E_{d}=10 \mathrm{TeV}$.

and has a well detectable spectral shape because of the sharp drop in the $\left(e^{+}+e^{-}\right)$flux at half the mass of the DM (see figure 3) due to a branching ratio of $40 \%$ for the eev channel, which shows a complementarity between the sensitivity of CALET and $\gamma$-ray measurements.

\section{Conclusion}

CALET, measuring the $\mathrm{TeV}$ region $\left(e^{+}+e^{-}\right)$spectrum directly for the first time with good energy resolution, will play an important role in indirect search of DM. In this context we investigated a decaying DM model where a fermionic DM decays to 2 charged and 1 neutral leptons. Despite strong constraint from diffuse $\gamma$-ray measurements, there exists a notable possibility of DM model to be able to explain the positron excess, when the the DM mass is around $1 \mathrm{TeV}$ and decays to $e e v$ and $\mu \mu v$ channel. We used 5 years simulated CALET data for the $\left(e^{+}+e^{-}\right)$spectrum to show that this DM decay signal can be well distinguished from a generic single pulsar source case. The 

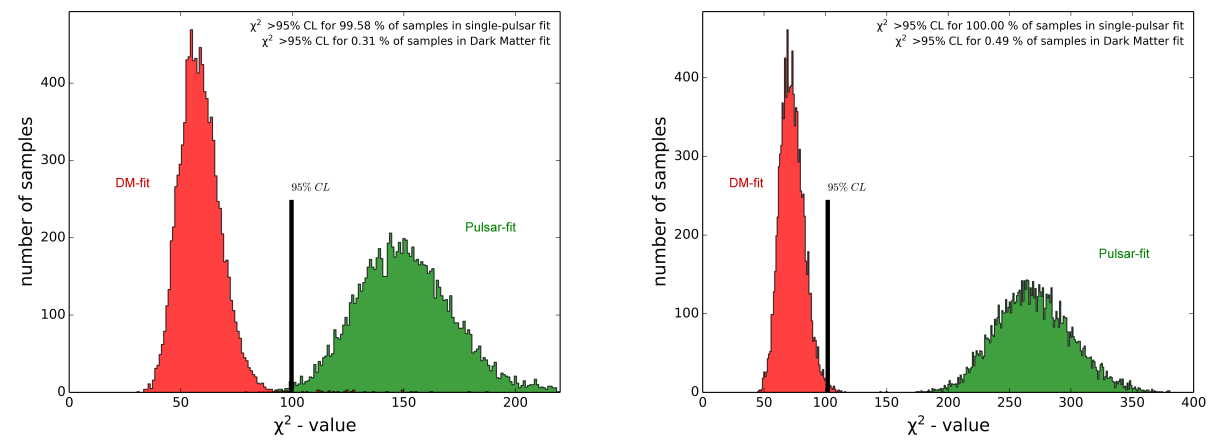

Figure 5: Left : $\chi^{2}$ distribution of fit of the single pulsar source to the simulated CALET data for $10^{3}$ DM samples + AMS-02 positron flux data (green curve) and re-fit of DM samples (red curve) using the same data points, for $2 \mathrm{TeV} \mathrm{DM}$ decay model with $E_{d}=2 \mathrm{TeV}$. Right : Same as figure on the left, but for the $1 \mathrm{TeV}$ DM model (decay excluding $\tau \tau \nu$ channel), with $E_{d}=10 \mathrm{TeV}$.

decay modes of the DM which are particularly compatible with the Fermi-LAT $\gamma$-ray measurement are possible to separate from the generic single pulsar spectrum with highest probability by the CALET expectations.

\section{Acknowledgements}

We would like to thank Dr. K.Kohri for valuable discussions about calculating the 3-particle decay spectra. S.B. is supported by JICA scholarship.

\section{References}

[1] L. Accardo, et al., Phys. Rev. Lett. 113, 121101 (2014).

[2] O. Adriani, et al., Nature 458, 607 (2009).

[3] P. D. Serpico, Astropart. Phys. 39-40, 2 (2012).

[4] M. Cirelli, M. Kadastik, M. Raidal, A. Strumia, Nucl. Phys. B813, 1 (2009).

[5] E. Nardi, F. Sannino, A. Strumia, JCAP 0901, 043 (2009).

[6] S. Torii, Proccedings of ICRC 2015 (2015), p. 581.

[7] Y. Asaoka, et al., Astropart. Phys. 91, 1 (2017).

[8] K. Kohri, N. Sahu, Phys. Rev. D88, 103001 (2013).

[9] M. Ackermann, et al., Astrophys. J. 750, 3 (2012).

[10] T. Sjostrand, S. Mrenna, P. Z. Skands, Comput. Phys. Commun. 178, 852 (2008).

[11] A. W. Strong, I. V. Moskalenko, Astrophys. J. 509, 212 (1998).

[12] J. F. Navarro, C. S. Frenk, S. D. White, Astrophys.J. 490, 493 (1997).

[13] M. Aguilar, et al., Phys. Rev. Lett. 113, 221102 (2014).

[14] M. Aguilar, et al., Phys. Rev. Lett. 113, 121102 (2014).

[15] B. Aschenbach, Nature 396, 141 (1998).

[16] K. Belotsky, R. Budaev, A. Kirillov, M. Laletin, JCAP 2017, 021 (2017).

[17] Y. Akaike, K. Kasahara, S. Torii, Proceedings of ICRC 2011 (2011), p. 371.

[18] Akaike.H, IEEE 19, 716 (Dec 1974). 